\title{
Development of Ultrasonic Axial Bolting Force Inspection System for Turbine Bolts in Thermal Power Plants*
}

\author{
Masataka SUDA**, Yasunori HASUO***, Akihiro KANAYA**, \\ Yukio OGURA****, Toshio TAKISHITA**** \\ and Yoshiaki SUZUKI****
}

\begin{abstract}
An ultrasonic axial bolting force inspection system is developed for the control of bolts used on turbine outercasings in a thermal power plant. The ultrasonic time of flight through the bolt under stress is analysed using acoustoelastic law. The proportional constant, conventionally called the material constant, is determined by an acoustoelastic constant $k$ and Young's modulus $E$ as $(1-k E)$. Measurements of $k$ for some kinds of steel bolts revealed that $k$ is approximately the same value $\left(-1.1 \times 10^{-11}\right.$ $\mathrm{m}^{2} / \mathrm{N}$ ) independent of heat treatment. Based on these results, the ultrasonic axial bolting force inspection system is developed. Applying this system to turbine bolts, it is found that the bolts could be controlled more precisely than ever.
\end{abstract}

Key Words: Nondestructive Inspection, Ultrasonic Inspection, Tensile Properties, Bolt Axial Force, Acoustoelastic Law

\section{Introduction}

The inner and outer casings of turbines in thermal power plants are so firmly bolted that they can be operated safely without any leakage of high-pressure steam. However, there is no definite method for the control of turbine bolts, except by measuring the elastic elongation of bolts after fastening with gauges. This tends to result in low work efficiency or imbalanced bolt fastening force. In addition, if bolts are fastened excessively for a long time, they might affect the life of the entire system, including that of the bolts.

Several instruments have been developed for measuring bolt fastening force (hereinafter, "axial

* Received 29th October, 1991. Paper No. 89-1414B

** Research Laboratory, Kyushu Electric Power Co., Inc. 2-1-47, Shiobaru, Minami-ku, Fukuoka 815, Japan

*** Kyuden Sangyo Co., Inc. 2-18-20, Najima, Higashiku, Fukuoka 813, Japan

**** FA Factory, Hitachi Construction Machinery Co., Ltd. 650, Kandatsu-machi, Tsuchiura, Ibaraki 300, Japan bolting force"), some of which are in practical use $\mathrm{e}^{(1),(2)}$. There are, however, no measuring instruments applicable to controlling the axial bolting force of a large number of long bolts, over $1 \mathrm{~m}$, such as turbine casing bolts. In addition, the axial bolting force of these bolts is so large that it is preferable to measure the bolts nondestructively without applying any torque to them.

Under these circumstances, we developed an ultrasonic axial bolting force tester, and applied it to nondestructive axial bolting force control and the prevention of uneven or excessive fastening of turbine casing bolts.

Applying axial bolting force to a bolt causes elastic elongation in the bolt, which also causes the velocity of ultrasonic waves passing in the bolt to decrease. It takes longer for the ultrasonic wave to travel through a loaded (fastened) bolt than a nonloaded bolt.

Consequently, the axial bolting force can be established by measuring the propagation time difference ${ }^{(3)}$.

To discuss the basic theory for measuring axial bolting force, we conducted an analysis employing the acoustoelastic constant ${ }^{(4)}$ and applied the results to the 
ultrasonic bolt axial bolting force tester, comparing the values obtained with actual values.

\section{Analysis with Bolt Model}

Using a bolt model as shown in Fig. 1, we conducted an analysis on ultrasonic propagation.

An ultrasonic wave is entered into the bolt from one end. It is reflected at the other end and returns. Its time of flight $t_{0}$ is given by Eq. ( 1 ), where $V_{0}$ is the wave velocity with no load. When the bolt is fastened, an axial bolting force is generated in the hatched portion of the figure.

$$
t_{o}=\frac{h+l_{1}+l_{2}+l_{3}+l_{4}}{V_{o}} \times 2
$$

The axial bolting force causes stresses in various parts of the bolt, which cause elastic elongation and variation in wave velocity.

We define the acoustoelastic constant $k$, which represents the wave velocity change ratio with stress $\sigma$, by Eq. ( 2 ).

$$
V_{\sigma}=V_{o}(1+k \sigma)
$$

where $V_{\sigma}$ is wave velocity with stress $\sigma$.

Time of flight $t_{F}$ with the applied axial bolting force is then given by Eq. ( 3 ),

$$
\begin{aligned}
t_{F} & =\left[\frac{h-0.4 D_{1}+l_{3}-0.4 D_{2}+l_{4}}{V_{o}}\right. \\
& +\frac{0.4 D_{1}+\Delta h+l_{1}+\Delta l_{1}}{V_{o} \cdot\left(1+k \cdot F / A_{1}\right)} \\
& \left.+\frac{l_{2}+\Delta l_{2}+\Delta l_{3}+0.4 D_{2}}{V_{o} \cdot\left(1+k \cdot F / A_{2}\right)}\right] \cdot 2
\end{aligned}
$$

where

$$
\begin{aligned}
& A_{1}=D_{1}^{2} \pi / 4 \\
& A_{2}=D_{2}^{2} \pi / 4 \\
& D_{2}=D_{1}-0.65 P \\
& \Delta h, \Delta l_{1} \sim \Delta l_{3}: \text { Elongation } \\
& F: \text { Axial bolting force } \\
& P: \text { Threaded portion. }
\end{aligned}
$$

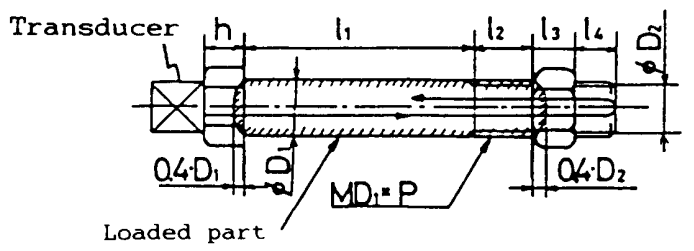

Fig. 1 Bolt model

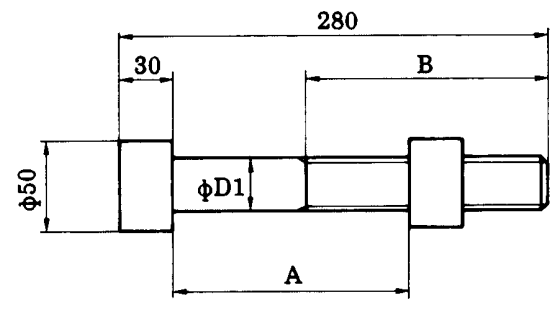

Fig. 2 Test bolt
In Eq. ( 3 ), elastic elongation and wave velocity variations are calculated for divided sections of the bolt as shown in Fig. 1 . A coefficient of 0.4 for the bolt head and threaded portion was obtained experimentally.

With Young's modulus and the acoustoelastic constant written as $E\left(\mathrm{~N} / \mathrm{m}^{2}\right)$ and $k\left(\mathrm{~m}^{2} / \mathrm{N}\right)$ respectively, the relation between the difference $\Delta t$ for time of flight without a load $t_{o}$ and time of flight with a load $t_{F}$, and axial bolting force $F$ is given by Eq. (4).

$$
\begin{aligned}
\Delta t & =t_{F}-t_{o}=F \cdot(1-k E) \\
& \times \frac{\left(0.4 D_{1}+l_{1}\right) / A_{1}+\left(0.4 D_{2}+l_{2}\right) / A_{2}}{2 \cdot V_{o} \cdot E}
\end{aligned}
$$

This reveals that axial bolting force $F$ can be obtained from the difference in time of flight $\Delta t$ using the proportional constant $(1-k E)$.

\section{Experimental Method}

Large-capacity turbine casing bolts have a diameter greater than $100 \mathrm{~mm}$ and length greater than $1 \mathrm{~m}$. We prepared test bolts of suitable lengths. Figure 2 shows the shape of the test bolts, in which $A: 105$ to $208 \mathrm{~mm}, B: 53$ to $204 \mathrm{~mm}, \phi D: 14$ to $20 \mathrm{~mm}$ and $P$ : 1.5 .

The materials of the test bolts were S $25 \mathrm{C}, \mathrm{S} 35$ $\mathrm{C}, \mathrm{S} 45 \mathrm{C}$ and S $50 \mathrm{C}$, heat-treated or not, and the materials for turbine casing bolts were $12 \mathrm{Cr}$ and $1 \mathrm{Cr}$.

The test device mainly consisted of load cells mounted on a hydraulic jack and a nut for holding the test bolt, as shown in Fig. 3 .

With an axial bolting force applied to the test bolt by the hydraulic jack in the test, the axial bolting force and the elongation were measured by the load cell and dial gauge, respectively.

The time of flight in the bolt was measured by the sing-around technique with an ultrasonic probe set on the bolt head. It was obtained from the difference between the reflecting waves in one round trip and two round trips, thus the time of flight in the probe was deducted.

The acoustoelastic constant $k$ of each test bolt was calculated by incorporating the applied axial bolting force $F$, time of flight for one round trip $t_{F}$ and

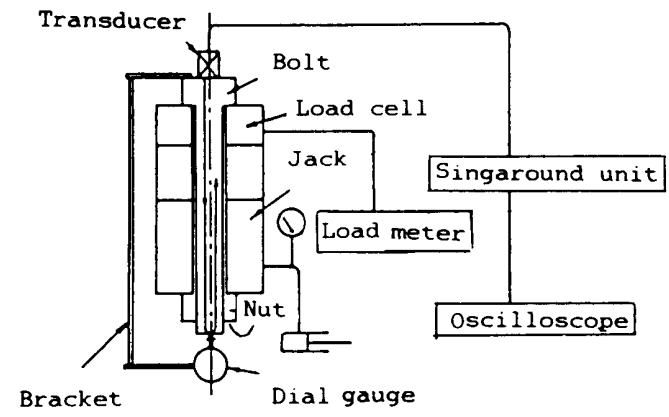

Fig. 3 Block diagram of the test device 
Young's modulus $E$ into Eq. ( 4 ).

\section{Experimental Results and Review}

Figure 4 shows the acoustoelastic constants $k$ 's of the test bolts.

The mean of the measured values was $-1.1 \times$ $10^{-11} \mathrm{~m}^{2} / \mathrm{N}$.

Figure 5 shows the calculated elongation of the bolt together with the measured values for comparison. There is good agreement, proving that the analy-

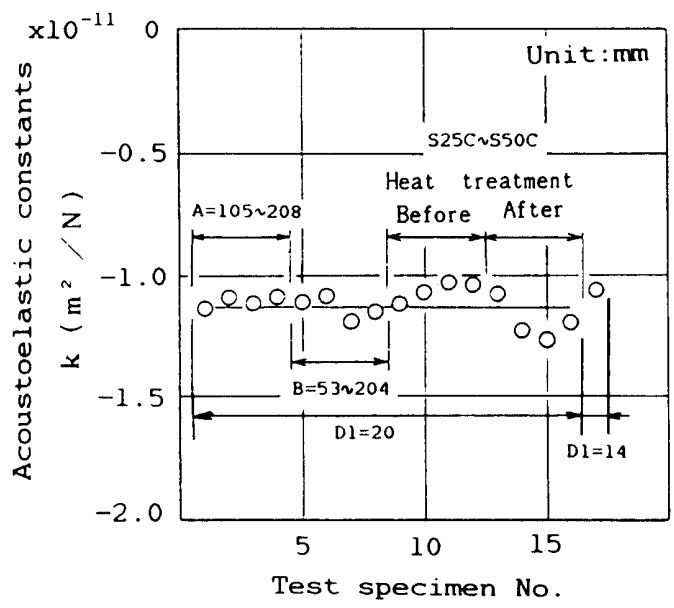

Fig. 4 Acoustoelastic constants of test pieces

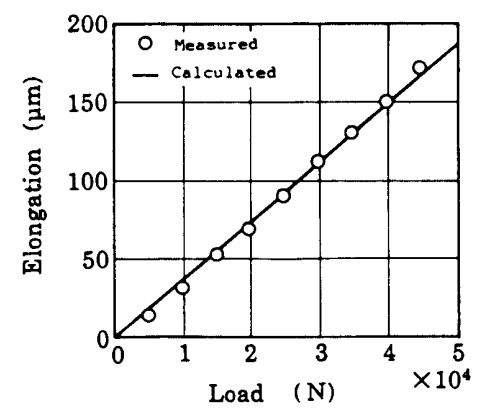

Fig. 5 Calculated and measured values of elongation (S $45 \mathrm{C})$

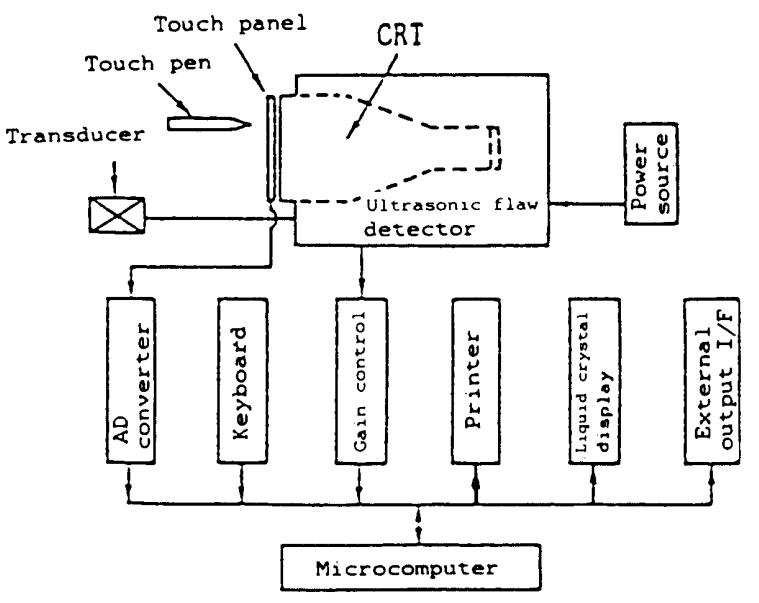

Fig. 6 Block diagram of the bolt axial-force tester sis used in the test represents the actual elongation very well.

The above results show that the coefficient ( 1 $-k E$ ) in Eq. ( 4 ) is nearly constant if the material is steel.

Consequently, the axial bolting force can be calculated from the dimensions of the bolt sections and the difference in time of flight $\Delta t$ between two cases with and without an applied axial bolting force.

Summarizing these results into software and combining it with an ultrasonic meter, we developed an axial bolting force tester.

Figure 6 shows a block diagram of the newly developed axial bolting force tester.

Figure 8 shows the results of measurement using the newly developed axial bolting force tester together with the Amsler's universal tester to apply axial bolting force as shown in Fig. 7. For the acoustoelestic constant $k$, the value of $-1.1 \times 10^{-11} \mathrm{~N} / \mathrm{m}^{2}$ was used. There is good agreement between the applied axial bolting force and the results of the measurements.

The newly developed axial bolting force tester was then employed to measure the axial bolting force of turbine casing bolts at a thermal power plant during periodic repairs.

Figures 9 and 10 show a turbine casing bolt and

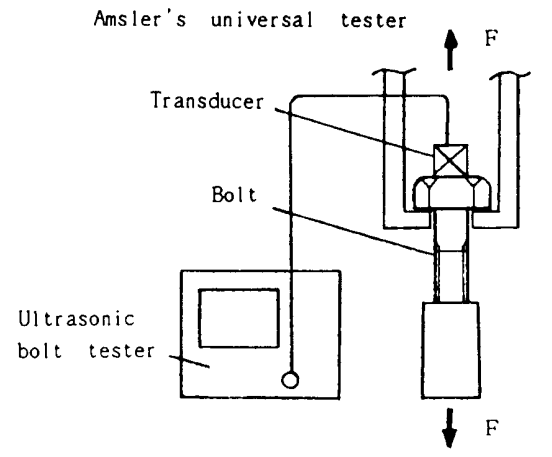

Fig. 7 Method for applying load to bolt

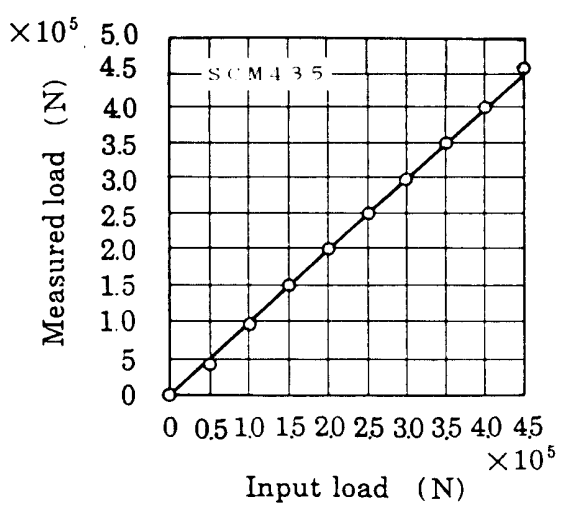

Fig. 8 Axial force measurement with Amsler's universal tester 


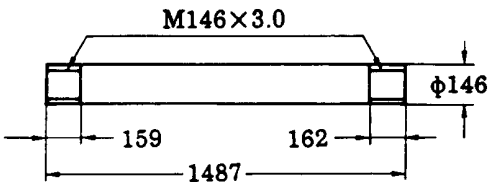

Material : Steel (12 Cr. V. Ta)

Fig. 9 Turbine bolt for thermal power plant

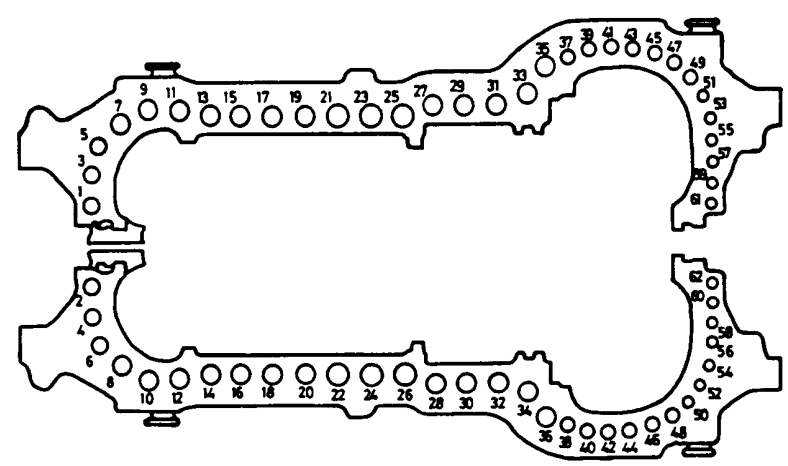

Fig. 10 Layout of turbine casing bolts

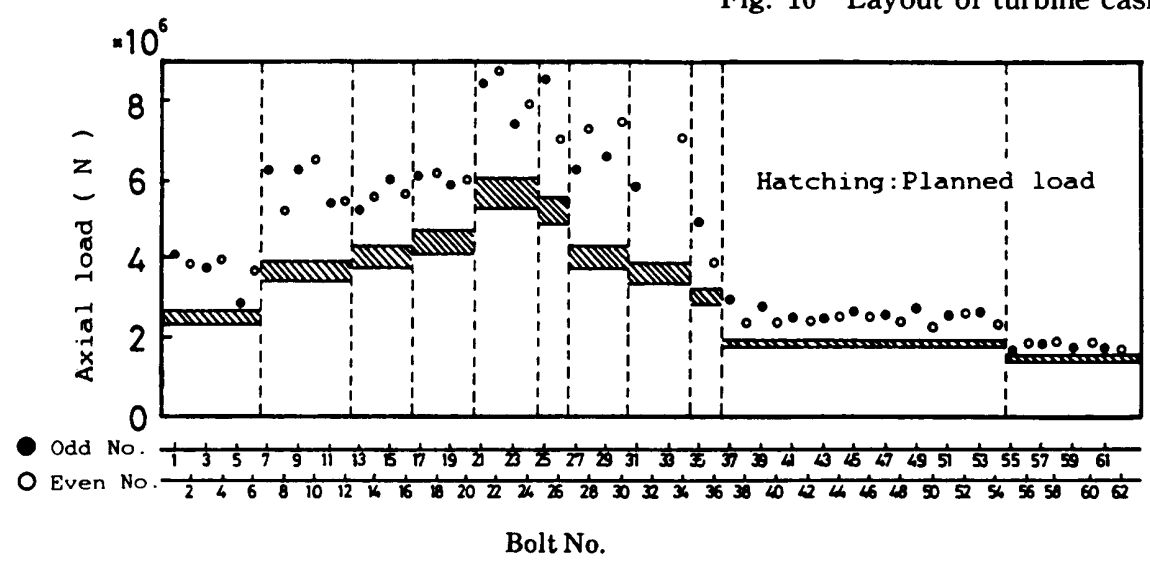

Fig. 11 Measurement results

the bolt layout, respectively.

Figure 11 shows one of the measurement results. The horizontal and vertical axes represent the bolt numbers and the measured axial bolting force, respectively.

The hatched portions represent each planned axial bolting force.

The figure reveals that the turbine casing bolts measured in the experiment were slightly overfastened, compared with the planned loads.

It is now possible to fasten all bolts with a suitable axial bolting force by controlling the fastening force with the present axial bolting force tester.

\section{Conclusions}

For the ultrasonic measurement of axial bolting force, we conducted an analysis of ultrasonic propagation on the basis of acoustoelastic law, obtaining the following results :

(1) Using the acoustoelastic constant $k\left(\mathrm{~m}^{2} / \mathrm{N}\right)$, we derived a bolt material constant $(1-k E)$ and proved that it was useful for measuring axial bolting force.

(2) Using several test samples of various materials (S $50 \mathrm{C}, \mathrm{S} 25 \mathrm{C}$ etc.) and shapes (effective length : 105 to $208 \mathrm{~mm}$, diameter : 14 to $20 \mathrm{~mm}$ ) and heat- treated or non-heat-treated materials, we measured the $k$ values, proving that they were nearly constant for all samples.

(3) We developed an axial bolting force tester using the constant $(1-k E)$ mentioned above, and employed it to measure the axial bolting force of turbine bolts at a thermal power plant. We found that conventional fastening was slightly excessive.

\section{References}

(1) Mori, T., Takekoshi, Y., Yagisawa, T., Ohyabu, M. and Nakamura, T., Measurement of Bolt Axial Force by Magnetic Technique, Trans. Jpn. Soc. Mech. Eng. (in Japanese), Vol. 47, No. 413, A (1980), p. 102.

(2) Makino, T., Sakai, T. and Toriyama, H., Development of the Bolt Clamping Force Measuring Device with Ultrasonic Waves, Toyota Engineering (in Japanese), Vol. 25, No. 1 (1975), p. 11.

(3) Yamamoto, E. and Motegi, R., Ultrasonic Sensors for Measuring Bolt Axial Stress (in Japanese), Jour. Jpn. Soc. Mech. Eng., Vol. 82, No. 731 (1979), p. 40.

(4) Fukuoka, H., Introduction to Acoustoelesticity (Part 1 Theory of Acoustoelasticity) (in Japanese), Jour. Jpn. Soc. NDI, Vol. 33, No. 9 (1984), p. 633. 